Site-bond percolation: a low-density series study of the uncorrelated limit

This article has been downloaded from IOPscience. Please scroll down to see the full text article.

1979 J. Phys. A: Math. Gen. 122073

(http://iopscience.iop.org/0305-4470/12/11/018)

View the table of contents for this issue, or go to the journal homepage for more

Download details:

IP Address: 129.74.250.206

The article was downloaded on 01/07/2010 at 15:23

Please note that terms and conditions apply. 


\title{
Site-bond percolation: a low-density series study of the uncorrelated limit $\dagger$
}

\author{
P Agrawal, S Redner, P J Reynolds, and H E Stanley \\ Center for Polymer Studies and Physics Department, Boston University, Boston, Mass. \\ 02215, USA
}

Received 7 November 1978

\begin{abstract}
A generalisation of the pure site and pure bond percolation problems is studied, in which both the sites and bonds are independently occupied at random. This generalisation-the site-bond problem-is of current interest because of its application to the phenomenon of polymer gelation. Motivated by considerations of cluster connectivity, we have defined two distinct models for site-bond percolation, models A and B. In model A, a cluster is considered to be a set of occupied bonds and sites in which the bonds are joined by occupied sites, and the sites are joined by occupied bonds. Since a bond cannot contribute to cluster connectivity if either site at its endpoints is not occupied, we define model B in which these 'non-connecting' bonds are treated as part of the cluster perimeter. We prove that the critical curve and critical exponents are the same for both models. For model B, we calculate low-density series expansions for the mean cluster size on the square lattice. We calculate three different series, using the following definitions of cluster size: site size, bond size, and a hybrid measure involving both site and bond size. All three series have been used to obtain the phase boundary between the percolating (gel) and non-percolating (sol) regions. Numerical evidence is presented which indicates that along the entire phase boundary the mean-size exponent $\gamma$ assumes a universal value.
\end{abstract}

\section{Introduction}

Recently, the percolation problem has attracted renewed interest (see e.g. the recent reviews of Stauffer (1979), Essam (1979) and Pfeuty and Guyon (1979)). One reason for this current interest is that it is becoming clear that generalisations of the pure percolation problem are likely to have extensive applications in the description of various phenomena in nature.

One generalisation of pure percolation that very recently has been used to describe both polyfunctional condensation and cross-linking of linear polymers (Coniglio et al 1979) is correlated 'site-bond' percolation. In this model, bonds represent chemical bonds, occupied sites represent monomers, and empty sites represent solvent molecules. Sites are correlated as in a lattice gas model of a binary mixture. Thus far, this model has been solved only on the Bethe lattice.

We have begun a study of site-bond percolation for more realistic lattices, and as an important first step we consider the uncorrelated model on the square lattice with both the sites and bonds independently occupied at random. This model was first mentioned by Frisch and Hammersley (1963), but it was not until 15 years later that the first calculations were performed, using Monte-Carlo methods (Hoshen 1978).

† Work supported in part by the ARO and AFOSR. 
In this work we present the first treatment of site-bond percolation using series methods. In $\S 2$ we first define the previously treated site-bond model, and then introduce a new model which appears to treat cluster connectivity in a more natural fashion. In $\S 3$ we outline the details of the cluster enumeration procedure. From the cluster data we calculate the mean-size function in a low-density series. Some important properties of these series are described in $\S 4$. Finally, in $\S 5$ we obtain the phase boundary separating the percolating (gel) and non-percolating (sol) species in the probability parameter space (cf figure 1) with an accuracy of $1-2 \%$ by using series analysis methods. We further make an approximate estimate for the critical exponent for the mean cluster size, and our analysis indicates that it assumes the universal two-dimensional value of approximately 2.43 along the entire phase boundary. In the Appendix we show that the two models introduced in $\S 2$ have the same phase boundary and the same critical exponents.

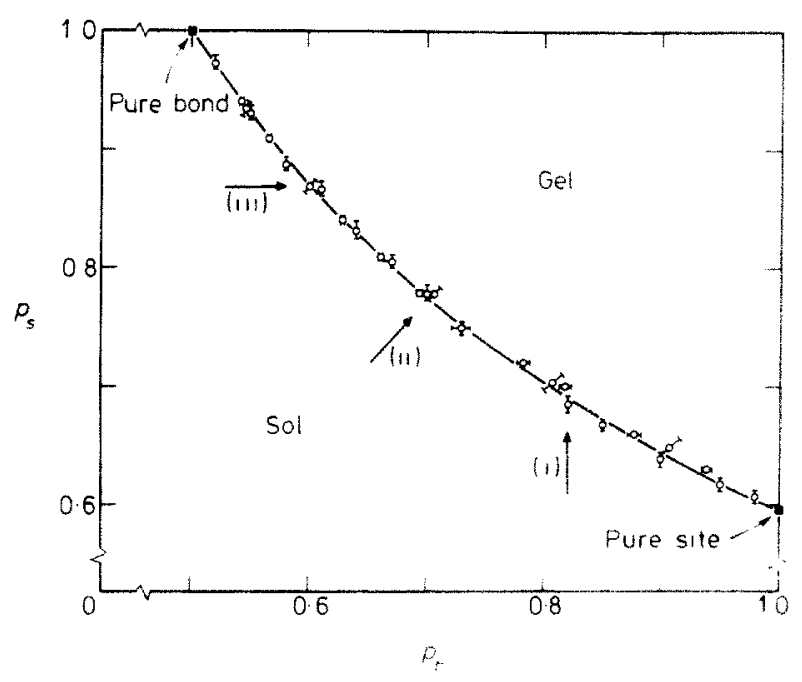

Figure 1. The phase diagram of the site-bond system in the $p_{s}-p_{b}$ parameter space. The critical curve, shown as a full curve, separates the percolating region, in which a gel has formed, and the non-percolating region, the sol phase. The error bars reflect subjective estimates of the reliability of the analysis methods employed in $\S 5$. The arrows indicate schematically how the various series probe the phase boundary.

\section{Two models of site-bond percolation}

\section{Model A}

The sites and bonds are randomly occupied with independent probabilities $p_{s}$ and $p_{b}$ respectively. Notice that the pure site problem is simply the limiting case $p_{b}=1\left(p_{s}\right.$ variable), while the pure bond problem is the limiting case $p_{s}=1$ ( $p_{b}$ variable). We define a cluster to be a set of occupied bonds and sites in which the bonds are joined by occupied sites and the sites are joined by occupied bonds.

It is clear that a bond cannot contribute to cluster connectivity if either or both endpoint sites are absent (cf figure 2). We call such bonds 'non-connecting'. From this consideration it is natural to exclude the non-connecting bonds from the cluster, and 


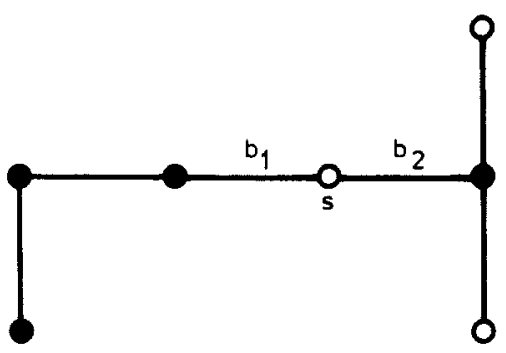

(a)

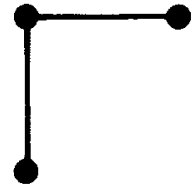

(b)

Figure 2. (a) Two typical clusters in model A. Full circles represent occupied sites and open circles represent unoccupied sites. The two clusters remain separate even though there exist bonds $\left(b_{1}\right.$ and $\left.b_{2}\right)$ from the two clusters which terminate at the same lattice site $s$. Such bonds are called non-connecting. $(b)$ The same two clusters in model $\mathrm{B}$ of site-bond percolation.

instead consider them to be in the cluster perimeter. We are thus led to define a second model.

\section{Model $B$}

Sites and bonds are occupied in the same way as in model A. However, we now define a cluster as a set of joined occupied sites and bonds, with the non-connecting bonds included as part of a generalised cluster perimeter. Notice that if we measure clusters by site size, then the non-connecting bonds have no effect on the size of a given cluster. However, if bond size is used, a cluster can have two different sizes in models A and B. In the next section we show how the inclusion of the non-connecting bonds in the cluster perimeter is incorporated into the low-density series calculation.

\section{Low-density series calculation for the mean cluster size}

The application of series expansions for percolation was first suggested by de Gennes et al (1959) and Domb (1959) and has been used extensively since then for studying many different systems (see e.g. Sykes et al (1976) and references therein). The series method is based upon exact enumeration of all possible clusters up to a size determined largely by the limits of computer capability. From the probability factors associated with each cluster, one may obtain power series for various macroscopic quantities, such as the mean cluster size. These series may then be extrapolated to obtain information about the percolation threshold.

To illustrate our computer enumeration and to show how the non-connecting bonds are treated as part of the cluster perimeter in model B we present the lowest few orders of the calculation in detail. In what follows it is most useful to measure clusters in terms of both their site and bond size; therefore we calculate the mean number of clusters of $s$ sites and $b$ bonds per site, $\left\langle n_{s, b}\right\rangle$.

To begin, we consider all clusters containing only one site. In model $A$ these clusters contain between zero and four bonds, all of which are non-connecting (cf figure 3 ). In model B such clusters contain one site and no bonds, and the non-connecting bonds are included in the perimeter as follows: If a cluster is to contain only one site, each perimeter site must be either unoccupied (this occurs with probability $q_{s}=1-p_{s}$ ), or 


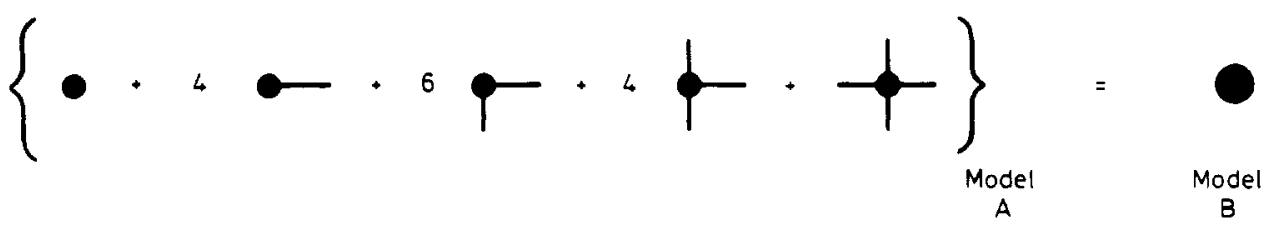

Figure 3. The one-site clusters. In model A, each cluster of $k$ non-connecting bonds may be embedded in $4 ! / k !(k-4)$ ! ways on the square lattice. If we include all such bonds in the cluster perimeter, we obtain the one-site, zero-bond cluster of model $B$.

else it is occupied but the bond between the perimeter site and the occupied site is absent (this occurs with probability $p_{s} q_{b}$, where $q_{b}=1-p_{b}$ ). Since the two possibilities are mutually exclusive, we obtain the factor $\left(q_{s}+p_{s} q_{b}\right)$ for each of the four perimeter sites $\dagger$. Implicit in the former case is the fact that a non-connecting bond may be present without altering the cluster. Hence we have

$$
\left\langle n_{s=1, b=0}\right\rangle=p_{s}\left(q_{s}+p_{s} q_{b}\right)^{4} .
$$

If the quantity in parentheses were expanded, the various terms obtained would represent the 16 clusters indicated in figure 3 . This illustrates that, for each cluster in model $\mathrm{B}$, there is a multiplicity of clusters in model A, and thus for the latter model the series calculation is relatively more time-consuming. Moreover, because model B appears to treat cluster connectivity more naturally, and because of the equivalence of the two models (cf Appendix), we shall henceforth focus on model B.

The contribution to $\left\langle n_{2,1}\right\rangle$ is found by similar reasoning to that used above. We find

$$
\left\langle n_{2,1}\right\rangle=2 p_{s}^{2} p_{b}\left(q_{s}+p_{s} q_{b}\right)^{6} .
$$

The overall factor of two on the right-hand side occurs because there are two distinct embeddings of the two-site cluster on the square lattice.

At third order, an important new feature in the perimeter factor arises which is central to our calculation. Consider the three-site cluster shown in figure 4 . Six of the

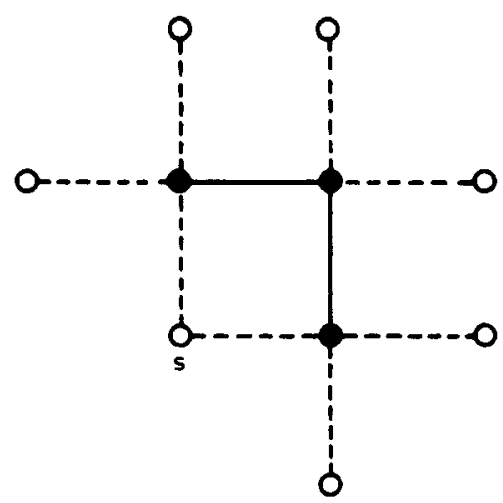

Figure 4. A three-site, two-bond cluster occurring in model B. Notice that six of the perimeter sites have valence 1 , in that they are nearest neighbour to only one cluster site, while the remaining perimeter site $s$ has valence 2 .

\footnotetext{
$\dagger$ Alternatively, we can obtain this factor noting that we wish to find [1 - (the probability that an occupied site is not isolated)]. This is simply $1-p_{s} p_{b}$. This line of reasoning becomes very complicated, however, when the perimeter site is nearest neighbour to more than one occupied site.
} 
perimeter sites can be joined to the cluster via a single non-connecting bond, and therefore we have for each of these sites a factor $q_{s}+p_{s} q_{b}$, as before. For the last perimeter site, however, there are two possible ways in which it may join to the cluster via non-connecting bonds. To exclude the possibility of both these connections requires a different perimeter factor, $q_{s}+p_{s} q_{b}^{2}$. In general, if a perimeter site is nearest neighbour to $k$ different cluster sites, a factor $q_{s}+p_{s} q_{b}^{k}$ occurs. We shall say that such a perimeter site has 'valence' $k$. Thus the enumeration of the $\left\langle n_{s, b}\right\rangle$ involves specifying the valences of all the perimeter sites.

In addition, we must also enumerate those configurations where two nearestneighbour occupied sites have a missing bond between them (cf figure 5), because each such missing bond contributes just a single factor of $q_{b}$ to the cluster perimeter. We shall refer to such bonds as 'empty', and denote the number of empties for each cluster by $e$.

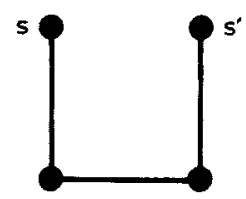

Figure 5. An example of an empty bond configuration in which there is a missing bond between sites $s$ and $s^{\prime}$. Notice that there are four distinct bond configurations possible for the given site configuration.

While a large amount of configurational information is required for each cluster, a simplification arises because the specification of the perimeter valences, and the number of empties for each cluster, is redundant. This may be seen by first noting that these variables are related to $b$ and to the number of independent closed loops $l$ in the following way:

$$
\sum_{k} k t_{k}=2(b+2)-4 l-2 e,
$$

where $t_{k}$ is the number of perimeter sites of valence $k$. To show this relation, note that $\Sigma k t_{k}$ is just the total number of non-connecting bonds in the cluster perimeter. This would be equal to $2(b+2)$ if the cluster had no closed loops or empty bonds. To obtain the term of $-4 l-2 e$, consider what happens when bonds are added to a cluster so that two distant sites are joined together to form a closed loop (cf figure 6). The addition of

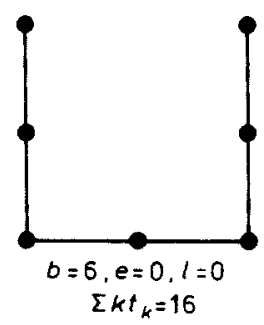

(a)

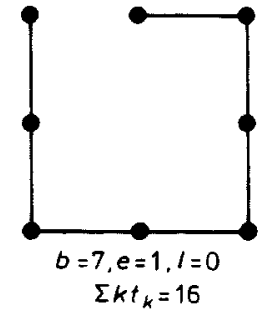

(b)

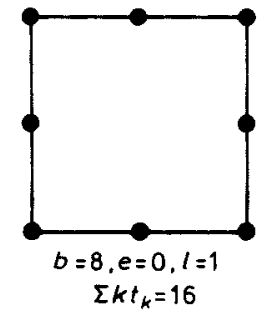

(c)

Figure 6. A demonstration of the relation $\Sigma_{k} k t_{k}=2(b+2)-4 l-2 e$. As bonds are added to the cluster in $(a)$ to form a closed loop, the total number of non-connecting bonds in the perimeter remains constant. 
an occupied bond increases the number of non-connecting bonds by two (assuming no empties are formed in the process), and thus the factor of $2 b$ in equation ( $3 a$ ). When the next-to-last bond is added, an empty bond is created, but now the number of non-connecting bonds, $\Sigma_{k} k t_{k}$, remains constant. Thus to balance this we require a factor -2 in $(3 a)$ for each empty bond. When the loop is closed, $\Sigma_{k} k t_{k}$ still remains constant, while $b$ increases by 1 and $e$ decreases by 1 . To compensate, we must subtract a factor 4 for each closed loop. Furthermore, if we use the fact that $l=(b+1-s)$, we obtain

$$
\sum_{k} k t_{k}=4 s-2 b-2 e .
$$

This shows that, for each cluster, specification of $e$ is indeed redundant once $s, b$ and $\Sigma_{k} k t_{k}$ have been calculated. By thus avoiding reference to $e$, we reduce the required computer memory by a factor of six, we save about $10 \%$ in computer time, and we achieve a considerable simplification in our counting algorithm.

From the techniques described by Martin (1974), we have developed computer programs to enumerate the clusters in site-bond percolation, and we have obtained all clusters of up to 12 bonds. (We note that, at 12 th order in bond size, clusters may contain between 9 and 13 sites.) From the configurational information, the mean-size series are calculated by a suitably weighted average of the $\left\langle n_{s, b}\right\rangle$. Two natural definitions of the mean-size function are ${ }^{\dagger}$

$$
S^{(s)}=\sum_{s, b} s^{2}\left\langle n_{s, b}\right\rangle
$$

and

$$
S^{(b)}=\sum_{s, b} b^{2}\left\langle n_{s, b}\right\rangle
$$

Since both the number of sites and the number of bonds measure cluster size, we also consider a 'hybrid' mean-size function

$$
S^{(s b)}=\sum_{s, b} s b\left\langle n_{s, b}\right\rangle
$$

Each of these series is then extended by one order in $p_{b}$ by a generalisation of the procedure described in Sykes and Glen (1976). This is accomplished by first calculating the low-density series for $\sum_{s, b} s\left\langle n_{s, b}\right\rangle$ and $\Sigma_{s, b} b\left\langle n_{s, b}\right\rangle$, and then exploiting the sum rules

$$
\sum_{s, b} s\left\langle n_{s, b}\right\rangle=p_{s}
$$

and

$$
\sum_{s, b} b\left\langle n_{s, b}\right\rangle=2 p_{s}^{2} p_{b}
$$

Notice that equation $(5 b)$ contains an overall factor of two because there are two ways in which a single bond may be embedded on the square lattice. The factor of $p_{s}^{2}$ occurs because in model B there must be an occupied site at both endpoints of each occupied bond. In addition, with the aid of the sum rules (5), we are able to enumerate by hand

$\dagger$ We have used the un-normalised form of the mean-size series. This definition will be more convenient for the discussion in the next section. 
the remaining relatively small number of 11 -site clusters not yet accounted for by the computer, thus extending the series for $S$ by one order in $p_{s}$. Therefore we have calculated the mean-size series to 13 th order in $p_{b}$ and 11 th order in $p_{s}$. The coefficients are shown in tables 1,2 and 3.

\section{Checks on the series coefficients}

In table 1 , if we let $p_{b}=1$, the entries reduce to the pure site series; similarly in table 2 , if we let $p_{s}=1$, we obtain the pure bond series. This is as we expect and is a reassuring check. Furthermore, we have the remarkable property that for all three series the coefficients of $p_{b}^{n} p_{s}^{n+1}$ are identical, and in fact are equal to the number of $n$-step self-avoiding walks (SAW's). This feature is most readily verified by employing the

Table 1. The coefficients in the series for the mean cluster size defined by the relation $S^{(s)}=\Sigma_{s, b} s^{2}\left\langle n_{s, b}\right\rangle$.

$$
\begin{aligned}
S^{(s)}= & p_{s} \\
& +p_{b}\left(4 p_{s}^{2}\right) \\
& +p_{b}^{2}\left(12 p_{s}^{3}\right) \\
& +p_{b}^{3}\left(36 p_{s}^{4}\right) \\
& +p_{b}^{4} p_{s}^{4}\left(100 p_{s}-12\right) \\
& +p_{b}^{5} p_{s}^{5}\left(284 p_{s}-48\right) \\
& +p_{b}^{6} p_{s}^{6}\left(780 p_{s}-252\right) \\
& +p_{b}^{7} p_{s}^{6}\left(2172 p_{s}^{2}-856 p_{s}+76\right) \\
& +p_{b s}^{8} p_{s}^{7}\left(5916 p_{s}^{2}-3388 p_{s}+300\right) \\
& +p_{b}^{9} p_{s}^{8}\left(16268 p_{s}^{2}-10832 p_{s}+2172\right) \\
& +p_{b}^{10} p_{s}^{8}\left(44100 p_{s}^{3}-37584 p_{s}^{2}+8340 p_{s}-544\right) \\
& +p_{b}^{11} p_{s}^{9}\left(120292 p_{s}^{3}-116296 p_{s}^{2}+38232 p_{s}-2880\right) \\
& +p_{b}^{12} p_{s}^{9}\left(324932 p_{s}^{4}-376220 p_{s}^{3}+140316 p_{s}^{2}-19624 p_{s}+300\right) \\
& +p_{b}^{13} p_{s}^{10}\left(881500 p_{s}^{4}-1135840 p_{s}^{3}+540468 p_{s}^{2}-92744 p_{s}+4236\right) \\
& +p_{b}^{14} p_{s}^{11}(\ldots+32400) \\
& +p_{b}^{15} p_{s}^{11}(\cdots-4624)
\end{aligned}
$$

Table 2. The coefficients in the series for the mean cluster size defined by the relation $S^{(b)}=\Sigma_{s, b} b^{2}\left\langle n_{s, b}\right\rangle$.

$$
\begin{aligned}
\hline S^{(b)}= & p_{b}\left(2 p_{s}^{2}\right) \\
& +p_{b}^{2}\left(12 p_{s}^{3}\right) \\
& +p_{b}^{3}\left(36 p_{s}^{4}\right) \\
& +p_{b}^{4} p_{s}^{4}\left(100 p_{s}-4\right) \\
& +p_{b}^{s} p_{s}^{5}\left(284 p_{s}-32\right) \\
& +p_{b}^{6} p_{s}^{6}\left(780 p_{s}-180\right) \\
& +p_{b}^{7} p_{s}^{6}\left(2172 p_{s}^{2}-688 p_{s}+40\right) \\
& +p_{b}^{8} p_{s}^{7}\left(5916 p_{s}^{2}-2792 p_{s}+208\right) \\
& +p_{b}^{9} p_{s}^{8}\left(16268 p_{s}^{2}-9352 p_{s}+1516\right) \\
& +p_{b}^{10} p_{s}^{8}\left(44100 p_{s}^{3}-32880 p_{s}^{2}+6432 p_{s}-316\right) \\
& +p_{b}^{11} p_{s}^{9}\left(120292 p_{s}^{3}-104288 p_{s}^{2}+29972 p_{s}-2000\right) \\
& +p_{b}^{12} p_{s}^{9}\left(324932 p_{s}^{4}-340276 p_{s}^{3}+115140 p_{s}^{2}-13864 p_{s}+184\right) \\
& +p_{b}^{13} p_{s}^{10}\left(881500 p_{s}^{4}-1042544 p_{s}^{3}+450148 p_{s}^{2}-70128 p_{s}+2688\right) \\
& +p_{b}^{14} p_{s}^{11}(\cdots+22528) \\
& +p_{b}^{15} p_{s}^{11}(\cdots-2896) \\
&
\end{aligned}
$$


Table 3. The coefficients in the series for the mean cluster size defined by the relation $S^{(s b)}=\Sigma_{s, b} s b\left\langle n_{s, b}\right\rangle$.

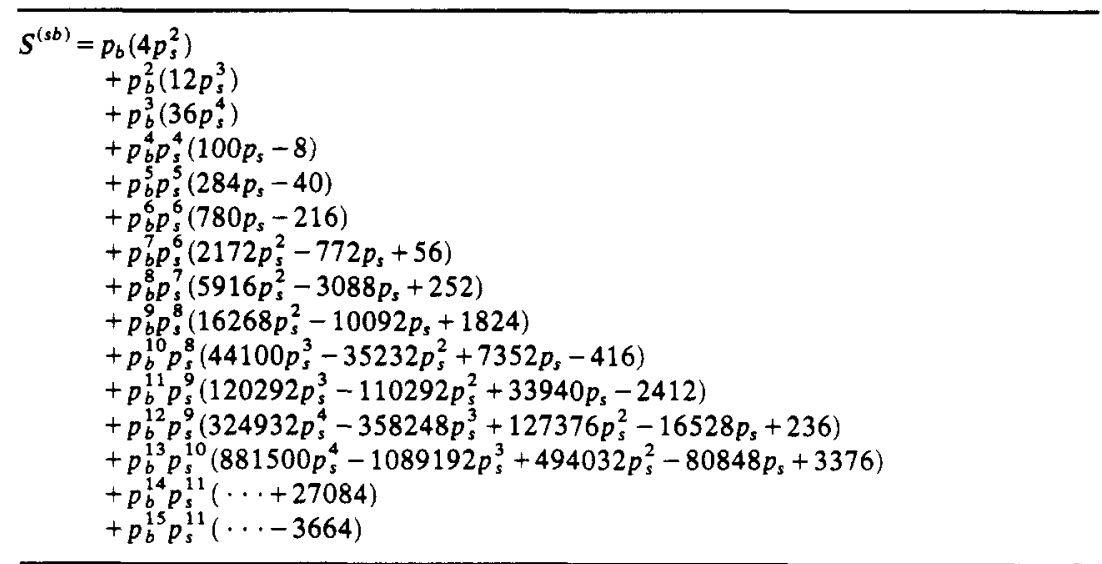

analogue of the fluctuation-dissipation theorem for percolation, in which the mean size may be expressed in terms of the pair-connectedness function (Essam 1971, Levinshtein et al 1975). With the aid of this theorem, it is relatively straightforward to verify the SAW property for $S^{(s)}$ and $S^{(b)}$ (cf figure 7 ). However, to treat $S^{(s b)}$ it is most convenient to first transform the model in the following way: We add sites, which may be occupied with probability $p_{b}$, at the midpoints of each bond, and we now consider all the bonds to be occupied. The resulting decorated site model is completely equivalent to site-bond percolation. (The existence of such a mapping was first mentioned in Frisch and Hammersley (1963).) On the decorated lattice, cluster size is most naturally measured by the total number of sites: these include sites at vertices ( $s$ on the original lattice) and decorated sites ( $b$ on the original lattice). Hence a natural definition of the mean cluster size is

$$
S^{(s+b)}=\sum_{s, b}(s+b)^{2}\left\langle n_{s, b}\right\rangle .
$$

With this definition, it now follows that between two fixed vertex sites the contribution at order $p_{b}^{n} p_{s}^{n+1}$ to the pair-connectedness is again due to SAw's only (notice that decorated sites may not be the endpoint of a SAW in model B of site-bond percolation). Since the SAW property for $S^{(s)}$ and $S^{(b)}$ was verified separately, it follows that $S^{(s b)}$ also has the SAW property.

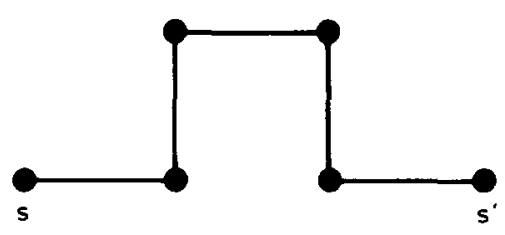

(a)

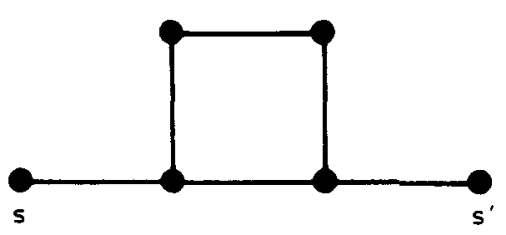

(b)

Figure 7. Two typical graphical contributions to the pair-connectedness between sites $\mathrm{s}$ and $s^{\prime}$. Only the SAW graph of $(a)$ contibutes at order $p_{b}^{n} p_{s}^{n+1}$. 


\section{Series analysis}

Our objective is to obtain the critical curve which divides the percolating and nonpercolating regions in the $p_{s}-p_{b}$ parameter space. We further wish to investigate whether the mean-size exponent $\gamma$ defined by $S \sim\left|p-p_{\mathrm{c}}\right|^{-\gamma}$ remains constant along this curve, in accord with universality. Here $S$ is any of the previously defined mean-size functions, $p$ means $p_{s}$ or $p_{b}$ or a linear combination of the two depending on the direction of approach to the critical curve (see below), and $p_{c}$ is the critical value of $p$.

To check the internal consistency of our analysis methods, and to aid in the estimation of the uncertainties in determining the critical curve, we have analysed all three of our mean-size series $(4 a)-(4 c)$. Furthermore, since each series is a function of both $p_{s}$ and $p_{b}$, we are able to probe the critical curve from any direction in this parameter space. We have chosen three directions of approach in our study: (i) fix $p_{b}$ and estimate the critical value of $p_{s}$; (ii) fix $p_{s}$ and estimate the critical value of $p_{b}$; and (iii) substitute $p_{s}=x p_{b}$ and find the critical value of $p_{b}$. These three choices correspond to approaching the critical curve vertically, horizontally or along a ray from the origin (cf figure 1). We have found it quite useful to employ all three directions of approach, because the best results seem to be obtained when the critical curve is probed roughly perpendicularly. Thus we have a total of nine different series for our analysis (three counting methods times three directions of approach).

In general, when we compare the analysis of the three series obtained by the different counting methods with the analysis of a single series probing the phase boundary from different directions the former case gives more consistent results. Thus we use the latter case for establishing an estimate of our confidence limits.

To map out the critical curve, we have used a variation of the technique introduced by Sykes et al (1976). Padé approximants to the logarithmic derivative series for $S$ are formed, and the positive real-valued roots and residues are selected. When the values of the residues are plotted against the corresponding location of the root, a single relatively smooth curve is usually defined (cf figure $8(a)$ ). Sykes et al have used this, together with the fact that there exists a complementary lattice (the matching lattice for site models and the dual lattice for bond models) which has its critical point at $1-p_{\mathrm{c}}$ of the original lattice. Thus if the residues obtained in the two related problems are plotted on complementary probability scales, the two curves intersect at $p_{\mathrm{c}}$. This intersection point may then be used to determine the value of $\gamma$. In this way Sykes et al have found numerically that $\gamma$ appears to be a dimensional invariant, in accord with universality.

For the site-bond problem, the definition of a complementary lattice is ambiguous, however, because the dual and match of the square lattice are not identical. Since it is not possible to derive series for the complement to the site-bond problem, we have found it necessary, in our application of the root-residue plot, to first assume a value for $\gamma$ to find $p_{c}$, and later test the validity of this assumption. We therefore provisionally assume that $\gamma$ equals the two-dimensional value of approximately 2.43 (Sykes et al 1976) along the entire crtical curve, for the following reasons: At the two endpoints, $\left(p_{s}=0.593, p_{b}=1\right)$ and $\left(p_{s}=1, p_{b}=0.5\right)$, we have the well-established numerical evidence of Sykes et al, based on longer series from pure percolation on the square lattice, that $\gamma=2.40 \pm 0.06$ and $\gamma=2.425 \pm 0.005$ respectively. Furthermore, for $p_{s}=p_{b}$ the model can be transformed to the previously discussed site problem on the decorated square lattice, where we again expect that $\gamma \simeq 2.43$ by universality. In addition, the smoothness postulate of Griffiths (1970) is directly applicable to the 

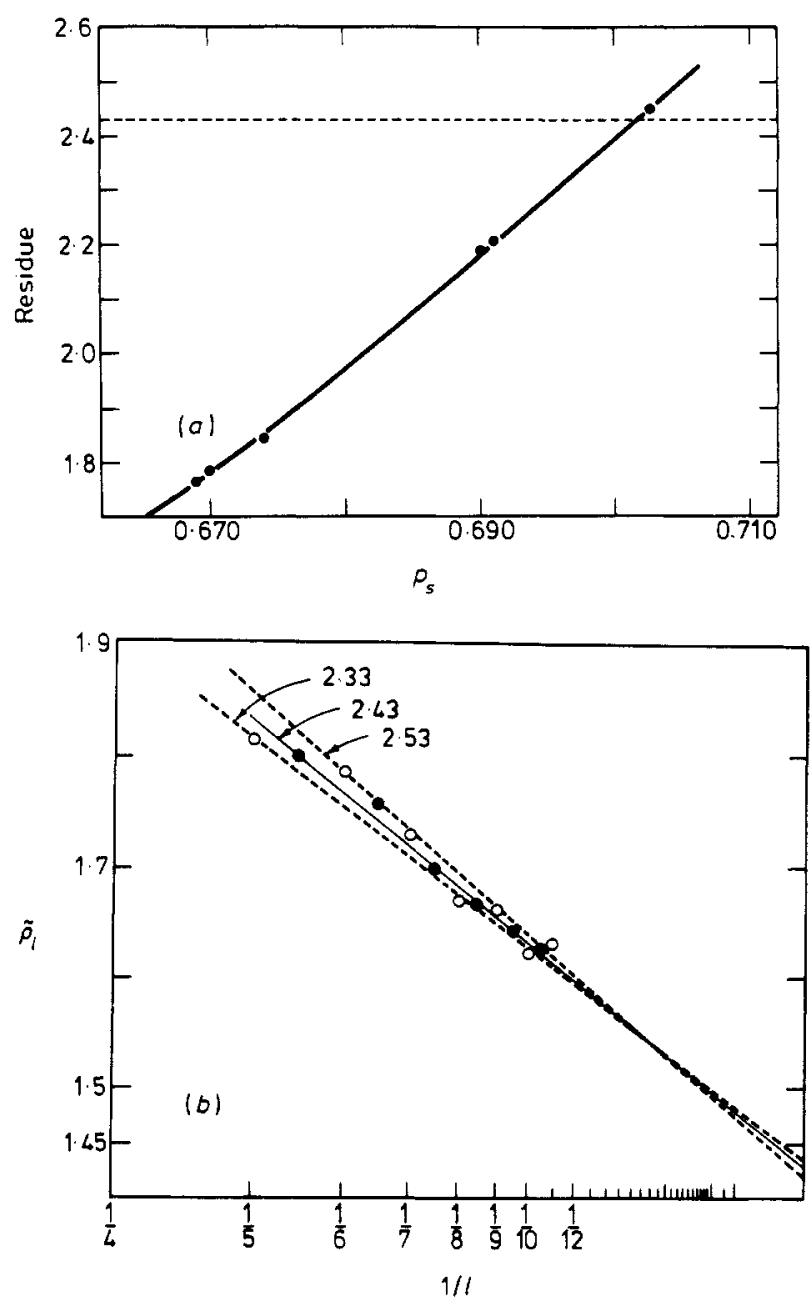

Figure 8. (a) A representative plot of the residue against the location of the corresponding root for the Padé approximants to the logarithmic derivative series for $S$. The case shown is that of $p_{s}$ variable, $p_{b}=0.8$ (vertical direction of approach) and site counting. If we provisionally assume that $\gamma=2.43$, then $p_{\mathrm{c}}$ is determined to be 0.702 . (b) Dependence of the 'square root' ratios $\dot{\rho}_{l}=\left(a_{l} / a_{l-2}\right)^{1 / 2}$ on $1 / l$ (open circles). The case of $p_{s}$ variable, $p_{b}=0.8$ and site counting is again shown. As a guide for the eye, two successive ratios are averaged (full circles). The full line indicates the expected asymptotic behaviour of the ratios when we use the values $\gamma=2.43, p_{c}=0.702$, as determined from $(a)$. The broken lines show the expected asymptotic behaviour when the values $\gamma=2.53, p_{\mathrm{c}}=0.697$ and $\gamma=2.33, p_{\mathrm{c}}=0.704$ from $(a)$ are used. Since the ratio data lie within the wedge-shaped region defined by the broken lines, we deduce that $\gamma=2 \cdot 43 \neq 0 \cdot 10$. Similar plots at other points along the phase toundary yield the conclusion that $\gamma$ remains 2.43 to within approximately $10 \%$.

site-bond model, and it provides further justification for the universality of $\gamma$. Finally, one can formulate a position-space renormalisation group for this model, following Reynolds et al (1977). It is found that the entire critical curve (including the pure site and pure bond endpoints) is controlled by a single fixed point, thus leading to universality along the phase boundary (Nakanishi and Reynolds 1979). 
From our analysis we have obtained the following behaviour for the three directions of approach considered: (i) Vertical ( fixed $p_{b}$ ). This series gives smooth, well-defined curves of root against residue along the entire phase boundary. However, the internal consistency of our analysis becomes worse as the pure site limit is approached. Nevertheless, we find that this direction of approach gives the best results although the series used here are of the shortest length. (ii) Horizontal (fixed $p_{s}$ ). This series again gives good results near the pure bond limit, and less internally consistent results toward the pure site limit. For $p_{s} \leqslant 0.75$ the series probe the phase boundary almost tangentially; this appears to be the source of the unreliability of our results for this case. (iii) Rays (setting $p_{s}=x p_{b}$ ). This direction of approach also seems to be quite useful, but once again mostly for $p_{s} \geqslant 0.75$, for the same reasons as in (ii).

On the whole, we find that the nine different series give results that are in good agreement with each other, especially away from the pure site limit. Near the pure bond limit we estimate $p_{\mathrm{c}}$ to $1 \%$ accuracy, while near the site limit the accuracy is about $2 \%$. The phase boundary thus obtained is shown in figure 1, with approximate error bars for each direction of approach.

Thus far, we have assumed the universality of $\gamma$ to obtain the critical curve. We test this assumption by considering the 'square root' ratios $\tilde{\rho}_{l}=\left(a_{l} / a_{l-2}\right)^{1 / 2}$, where $a_{l}$ is the $l$ th coefficient in the mean-size series. When the $\tilde{\rho}_{l}$ are plotted against $1 / l$, in the limit $l \rightarrow \infty$ the points should converge to $p_{\mathrm{c}}^{-1}$ asymptotically, forming a straight line of slope $p_{c}^{-1}(\gamma-1)$. This ideal behaviour does not occur on the square lattice, however, because of the 'antiferromagnetic' singularity in the series (see e.g. Gaunt and Guttmann 1974). This singularity, on the negative $p$ axis, gives rise to the characteristic even-odd oscillations in the ratios. Thus a limiting value of the slope is not readily obtained, unless we use some averaging procedure for the ratio data. While the estimates obtained for critical behaviour are not conclusive, they can be used in conjunction with the root-residue curves of the type shown in figure $8(a)$ to yield the following important consistency check: Each point on the root-residue curve determines a pair $\left(p_{c}, \gamma\right)$, from which we may specify a straight line of slope $p_{c}^{-1}(\gamma-1)$ and intercept $p_{c}^{-1}$ on a $1 / l$ ratio plot by assuming the asymptotic form of $\tilde{\rho}_{l} \sim p_{c}^{-1}[1-(\gamma-1) / l]$. From the line which best fits the ratio data subjectively, we then infer a value for $\gamma$.

This approach works quite well for both the horizontal and vertical series, but not for the ray series, where the ratios do not exhibit the simple even-odd oscillations on a $1 / l$ plot. Thus, with the exception of this last series, we have found that the value of $\gamma$ is consistent to $\pm 10 \%$ with the value 2.43 . This conclusion holds for almost the entire critical curve, except very near the site limit where all three series are very poorly behaved. This problem was expected, since the critical behaviour has not been determined precisely by series for the pure site problem, even though very lengthy series have been analysed (Sykes et al 1973). We thus conclude that, with the above provisos, $\gamma$ indeed appears universal.

\section{Summary}

We have considered the site-bond percolation problem, a model which may be useful in describing polymer gelation. We have introduced two specific models of site-bond percolation, models A and B. As a first step in understanding this system we have considered the non-interacting limit. We have calculated low-density series for the mean-size function $S\left(p_{s}, p_{b}\right)$ for model B. Series analysis has been used to calculate the 
phase diagram (cf figure 1) and to provide evidence that the exponent assumes a universal two-dimensional value of approximately 2.43 along the phase boundary separating the percolating (gel) and non-percolating (sol) species. Thus we have confirmed that for our uncorrelated model ihe exponents are the same as those for pure site and bond percolation. This supports the conjecture of Coniglio et al (1979) that percolation exponents should be expected for real gels close to the critical point.

\section{Acknowledgments}

We thank A Coniglio, W Klein and H Nakanishi for many very useful discussions in the course of this work. We also thank D Stauffer for helpful comments on the manuscript.

\section{Appendix. Equivalence of the models of site-bond percolation}

The two models introduced in $\S 2$ can be considered equivalent if we can show that: ( $a$ ) the critical curves are identical for both models, and $(b)$ the critical exponents are also identical.

Proof of $(a)$. We must show that, if there exists an infinite cluster in model A, then it necessarily implies the existence of an infinite cluster in model B, and vice versa. If site counting is used, then the definition of cluster size is identical for both models A and B, and the hypothesis is certainly true. Now consider bond counting. If there exists an infinite cluster in model B, then an infinite cluster in model A must have already formed, since in this case non-connecting bonds are now included in the definition of a cluster. To show the converse, note that the number of bonds belonging to the cluster in model $\mathrm{B}$ is always a finite fraction $f$ of the number of bonds in the cluster according to model $\mathrm{A}$ (except for the trivial case of the zero-bond, one-site cluster). It is readily verified that, on the square lattice, $N_{\mathrm{b}} /\left(3 N_{\mathrm{b}}+4\right) \leqslant f<1$, where $N_{\mathrm{b}}$ is the number of bonds in a cluster according to model $\mathrm{A}$. The lower bound, which is relevant to this proof, is determined by clusters with no empty bonds or closed loops. Thus the existence of an infinite cluster in model A implies the existence of an infinite cluster in model B, and hypothesis $(a)$ is proved.

Proof of $(b)$. Let $S_{\mathrm{A}}$ and $S_{\mathrm{B}}$ be the mean cluster size (with the same counting employed for both functions) for models $A$ and $B$ respectively. We assume that at the critical curve these functions diverge as

$$
S_{\mathrm{A}} \sim\left(p-p_{\mathrm{c}}\right)^{-\gamma_{\mathrm{A}}}
$$

and

$$
S_{\mathrm{B}} \sim\left(p-p_{\mathrm{c}}\right)^{-\gamma_{\mathrm{B}}},
$$

where $p$ means $p_{s}$ or $p_{b}$ or a linear combination of the two, depending on the direction of approach to the critical curve.

If site counting is employed, then in fact we have $S_{\mathrm{A}}=S_{\mathrm{B}}$. With bond counting we note that from the proof of (a) we have the large- $N$ inequality, $S_{\mathrm{A}}>S_{\mathrm{B}} \geqslant\left(S_{\mathrm{A}} / 3\right)$. Therefore $\gamma_{\mathrm{A}}=\gamma_{\mathrm{B}}$. 


\section{References}

Coniglio A, Stanley H E and Klein W 1979 Phys. Rev. Lett. 42 518-22

Domb C 1959 Nature, Lond. 184509

Essam J W 1971 J. Math. Phys. 12 874-82 1979 Preprint

Frisch H L and Hammersley J M 1963 J. Soc. Indust. Appl. Math. 11 894-918

de Gennes P G, P Lafore and J P Millot 1959 J. Chem. Phys. Solids 11 105-10

Gaunt D S and Guttman A J 1974 Phase Transitions and Critical Phenomena vol 3, ed. C Domb and M S Green (New York: Academic) pp 181-243

Griffiths R B 1970 Phys. Rev. Lett. 24 1479-82

Hoshen J 1978 Preprint

Levinshtein M E, Shklovskii B I, Shur M S and Efros A L 1975 Sov. Phys. -JETP 42 197-200

Martin J L 1974 Phase Transitions and Critical Phenomena vol 3, ed. C Domb and M S Green (New York: Academic) pp 97-112

Nakanishi H and Reynolds P J 1979 Phys. Lett. 71 A 252-4

Pfeuty $P$ and Guyon 1979 Preprint

Reynolds P J, Klein W and Stanley H E 1977 J. Phys. C: Solid St. Phys. 10 L167-72

Stauffer D 1979 Phys. Rep. 54 1-74

Sykes M F, Gaunt D S and Glen M 1976 J. Phys. A: Math. Gen. 9 97-103

Sykes M F and Glen M 1976 J. Phys. A: Math. Gen. 9 87-95

Sykes M F, Martin J L and Essam J W 1973 J. Phys. A: Math., Nucl. Gen. 6 1306-9 